\title{
Towards Personalized Imaging in Image-guided Radiotherapy
}

\author{
Yibao Zhang' and Jun Deng'* \\ ${ }^{1}$ Key Laboratory of Carcinogenesis and Translational Research (Ministry of Education) \\ Department of Radiotherapy, Peking University Cancer Hospital \& Institute, China \\ ${ }^{2}$ Department of Therapeutic Radiology, Yale University, USA
}

Received: December 07, 2013; Accepted: January 23, 2014; Published: January 24, 2014

"Corresponding author: Jun Deng, Department of Therapeutic Radiology, Yale University, 15 York Street, LL 508-Smilow, New Haven, CT 06510-3221, USA, Tel: 203-200-2013; Fax: 203-200-2054; E-mail: jun.deng@yale.edu

\begin{abstract}
A variety of radiological imaging modalities (e.g., CT, PET, CBCT, and EPID) are applied in the cancer radiotherapy for different clinical purposes, yet none of them are individually optimized to provide the patient with the best possible benefit/risk balance. Towards this goal, we propose to personalize the CT and CBCT scans in image-guided radiotherapy to maintain the benefit of radiological imaging while greatly reducing the ionization radiation exposure to the patient.

Keywords: IGRT; ALARA; Imaging dose; Cancer risk; Personalized imaging
\end{abstract}

\section{Abbreviations}

IGRT: image-guided radiotherapy; ALARA: as low as reasonably achievable; CT: computed tomography; PET: positron emission tomography; CBCT: cone beam computed tomography; kVCBCT: kilo-voltage cone beam computed tomography; MRI: magnetic resonance imaging; EPID: electronic portal imaging device; LINAC: linear accelerator; OAR: organ-at-risk

\section{Background}

Recently, with the advent of linear accelerators equipped with on-board x-ray images and advanced imaging technologies, image-guided radiotherapy (IGRT) has gained worldwide momentum in the radio therapeutic management of cancers in terms of volumetric information-based accurate tumor localization, real-time patient setup and margin reduction $[1,2]$. The involved technologies include digital radiography, computed tomography (CT), cone beam CT (CBCT), magnetic resonance imaging (MRI), positron emission tomography (PET), and megavoltage electronic portal imaging device (EPID) etc. [1] Figure 1 illustrates the three modalities that have been most widely used in IGRT, where CT is primarily used for treatment planning dose calculation while kVCBCT and EPID are mainly for tumor localization and patient setup. By focusing highly conformal tumoricidal doses to the cancerous tissues, higher tumor control with reduced normal tissue complication is expected.

Currently, the IGRT process has adopted a personalized treatment plan which is optimized to escalate the target dose yet protect the organs-at-risk (OARs) from any unnecessary exposure. However, the radiotherapy-associated radiological imaging is still conducted uniformly across the field worldwide regardless of the varieties of gender, age, ethnicity and body dimension. This 'one imaging modality/protocol fits all' practice may risk petite patients and children to unnecessary radiation dose without further benefiting them from the slightly increased image quality $[3,4]$. Hence, it is important to personalize the radiological imaging procedures used in IGRT in order to reduce the imaging doses and associated cancer risks without compromising patient care.

\section{Personalized imaging in IGRT}

In diagnostic radiology, the epidemiological risks associated with the radiation dose of diagnostic imaging have been repeatedly reported as an increasing public threat [5-7]; hence a lot of efforts have been made to optimize the radiation doses especially for the CT scan, which accounts for the largest portion of medical exposure today [8-10]. Approaches like the use of bowtie filter and automated exposure control are now established practices in diagnostic CT. More recently, the 'Image Gently' [11] and 'Image Wisely' [12] campaigns have been initiated to increase awareness of the opportunities to lower radiation doses in the imaging of children and adults.

However, the personalized imaging in the process of radiotherapy has not yet become a clinical routine. Relative to the large amount of treatment dose from high-energy mega-voltage photon beams, the imaging dose from kilo-voltage $x$-rays is much lower, up to a few percent $[13,14]$. Moreover, the image guidance for radiotherapy is relatively new technology compared to the long history of radiology [1]. It will take the clinicians and the general public some time to realize the necessity and develop the optimizers for low-dose imaging in radiotherapy.

In accordance with the 'Image Gently' and 'Image Wisely' campaigns, radiation exposure should always be operated under the principle of 'As Low As Reasonably Achievable' (ALARA). That is why we propose the concept of personalized imaging in IGRT, which means that CT and CBCT scan settings should no longer be site-specific or protocol-specific, rather patient-specific, with a balanced consideration of patient anatomy, gender, age, ethnicity, 


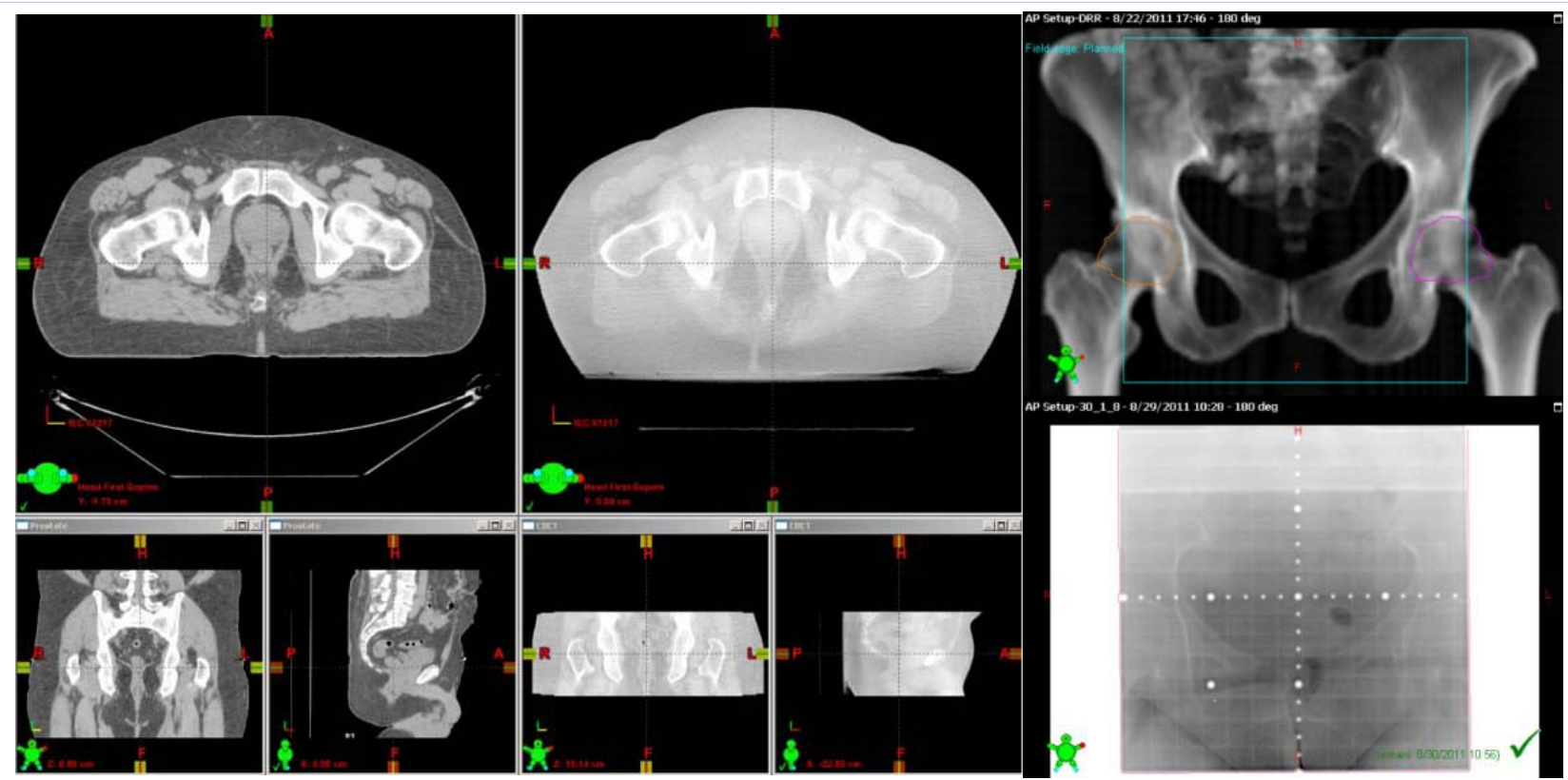

Figure 1: Three most frequently used imaging modalities in IGRT: CT (left), kVCBCT (middle), and EPID (right).

treatment history and treatment priority. These parameters would affect the quality of radiotherapy treatment, the total radiation exposure to the patient, and the potential secondary cancer risks [15].

In fact, there have been several strong reasons under scoring the significance of optimizing the imaging procedure for individual patient treated with radiotherapy. First of all, the image scanning covers a broader body region than the treatment field, which exposes OARs to ionizing irradiation without any shielding. Secondly, the imaging guidance is conducted repetitively due to the multi-fractionation of radiotherapy, which accumulates a considerable radiation dose to OARs. Thirdly, some imaging modalities applied in radiotherapy like CBCT deposit comparable or even higher dose per scan than the diagnostic CT $[13,14]$. Fourthly, most recipients of radiotherapy are cancer patients who might be genetically more vulnerable than general public to carcinogenesis like radiation [16], and the probability of stochastic effect is proportional to the radiation dose without a threshold [17]. Last but not least, a dramatic inter-personal dose disparity has been observed which put the petite patients especially children under higher risks [13].

\section{Personalized CT scans}

In North America, it is a clinical routine to reduce the $\mathrm{kVp}$ and $\mathrm{mAs}$ based on the body weight in the pediatric CT imaging to reduce the radiation dose $[18,19]$. The design of simulation CT used for radiotherapy is very similar to a diagnostic CT, except for its larger bore, a flat couch and some immobilization accessories. However, the simulation CT is not only used for diagnostic imaging, but also essential for the dose calculation by providing the electron density of the medium [20]. Although the correlation between the Hounsfield Unit (HU) and the density is subject to the scanning parameters [21], most treatment planning systems adopt single conversion table even if the planning CTuses different settings to scan various body regions [22]. More investigations would be needed to study the impact on the accuracy of electron density and dose calculation if $\mathrm{kVp}$ and $\mathrm{mAs}$ are changed dramatically as a result of pediatric parameter optimization for example.

\section{Personalized CBCT scans}

Our current practices of kVCBCT often involve multiple acquisitions of CBCT scans at default settings with scan parameters preset by the manufacturer for the selected scan protocol $[3,4,13,14]$. While a variety of site-specific scan protocols have been provided by the manufacturer aiming at reducing imaging doses in the patients, all those protocols are essentially non-personalized with no consideration of individual patient being scanned. With fixed settings applied to all the patients, over-exposure or under-exposure will undoubtedly occur $[13,14,23]$. While over-exposure inflicts unnecessary radiation on the patients, under-exposure leads to unacceptable image quality, none of which is ideal for clinical applications [24]. Although the users may adjust the preset scan parameters on-the-fly, it is essentially a trial-and-error process and often ends up with repeated scans with more doses deposited than intended. Therefore, it will be highly desirable to optimize the kVCBCT scans customized for individual patient.

As an approach to reduce the patient setup uncertainties, the image guidance in IGRT will not undermine the treatment delivery which has already provided a favorable flexibility of personalized optimization [1]. As one of the major contributors of imaging dose in IGRT, there is a considerable potential in reducing the unnecessary exposure to the petite patients by adjusting the scanning settings in CBCT $[3,4,13]$. Proper shielding or limiting the scan range into a reasonably small coverage will be very 
helpful in the protection of OARs [25]. When possible, replacing 3-dimentional CBCT scans with 2-dimentional imaging is a good choice. Restricted use of fluoroscopy is also recommended [26]. The choice of image modality and parameters should be evaluated carefully based on the clinical goal for the specific patient.

\section{Conclusion}

Any imaging optimization applied in the radiotherapy should consider the possible impact on the patient safety as well as the accuracy of the treatment plan delivery. A comprehensive yet balanced personalization including the choice of modalities and adjustment of scan settings is preferred. The various demands for the image quality depend on the specific clinical purposes such as bone marker registration, soft tissue monitoring, tumor tracking or treatment planning, which should be evaluated carefully before imaging optimization. By optimizing the image guidance in the IGRT, it is feasible and highly desirable to protect the patient from excessive radiation dose and cancer risk, while maintaining sufficient image quality for a specific clinical application.

\section{References}

1. Dawson LA, Sharpe MB (2006) Image-guided radiotherapy: rationale, benefits, and limitations. Lancet Oncol 7(10): 848-858

2. Marchant TE, Amer AM, Moore CJ (2008) Measurement of inter and intra fraction organ motion in radiotherapy using cone beam CT projection images. Phys Med Bio 53(4): 1087-1098.

3. Zhang Y, Yan Y, Nath R, Bao S, Deng J (2012) Personalized assessment of $\mathrm{kV}$ cone beam computed tomography doses in image-guided radiotherapy of pediatric cancer patients. Int J Radiat Oncol Biol Phys; 83(5): 1649-1654.

4. Zhang Y, Yan Y, Nath R, Bao S, Deng J (2012) Personalized estimation of dose to red bone marrow and the associated leukaemia risk attributable to pelvic kilo-voltage cone beam computed tomography scans in image-guided radiotherapy. Phys Med Biol 57(14): 45994612 .

5. Fazel R, Krumholz H, Wang Y, Ross JS, Chen J, et al. (2009) Exposure to Low-Dose Ionizing Radiation from Medical Imaging Procedures. N Engl J Med 361(9): 849-857.

6. Hall EJ, Brenner DJ (2008) Cancer risks from diagnostic radiology. $\mathrm{Br}$ J Radiol 81(965): 362-378.

7. Pearce MS, Salotti JA, Little MP, McHugh K, Lee C, et al. (2012) Radiation exposure from CT scans in childhood and subsequent risk of leukaemia and brain tumours: a retrospective cohort study. Lancet 380(9840): 499-505.

8. McCollough CH, Primak AN, Braun N, Kofler J, Yu L et al. (2009) Strategies for reducing radiation dose in CT. Radiol Clin North Am 47(1): 27-40.

9. Yu L, Liu X, Leng S, Kofler JM, Ramirez-Giraldo JC, et al. (2009) Radiation dose reduction in computed tomography: techniques and future perspective. Imaging Med 1(1): 65-84.

10. Wilting JE, Zwartkruis A, van Leeuwen MS, Timmer J, Kamphuis AG, et al. (2001) A rational approach to dose reduction in CT: individualized scan protocols. Eur Radiol 11(12): 2627-2632.

11. Goske MJ, Applegate KE, Boylan J, Butler PF, Callahan MJ, et al. (2008) The Image Gently campaign: Increasing CT radiation dose awareness through a national education and awareness program. Pediatr Radiol 38(3): 265-269.

12. Brink JA, Amis ES Jr (2010) Image wisely: A campaign to increase awareness about adult radiation protection. Radiology 257(3): 601602

13. Deng J, Chen Z, Roberts KB, Nath R (2012) Kilovoltage imaging doses in the radiotherapy of pediatric cancer patients. Int J Radiat Oncol Biol Phys 82(5): 1680-8.

14.Ding G X, Coffey C W (2009) Radiation dose from kilovoltage cone beam computed tomography in an image guided radiotherapy procedure. Int J Radiat Oncol Biol Phys 73(2): 610-617.

15. National Research Council (2006) Health risks from exposure to low levels of ionizing radiation: BEIR VII - Phase 2. Committee to Assess Health Risks from Exposure to Low Levels of Ionizing Radiation. Washington, DC: National Academies Press.

16. Hsu TC, Cherry LM, Samaan NA (1985) Differential mutagen susceptibility in cultured lymphocytes of normal individuals and cancer patients. Cancer Genet Cytogenet 17(4): 307-313.

17. Wagner LK, Eifel PJ, Geise RA (1994) Potential Biological Effects Following High X-ray Dose Interventional Procedures. J Vasc Interv Radiol 5(1): 71-84

18. Reid J et al. (2010) Optimization of $\mathrm{kVp}$ and $\mathrm{mAs}$ for pediatric lowdose simulated abdominal CT: is it best to base parameter selection on object circumference? AJR Am J Roentgenol 195 (4): 1015-1020.

19. Arch ME, Frush DP (2008) Pediatric body MDCT: A 5-year follow-up survey of scanning parameters used by pediatric radiologists. AJR Am J Roentgenol 191(2): 611-617.

20. Thomas SJ (1999) Relative electron density calibration of CT scanners for radiotherapy treatment planning. Br J Radiol 72(860): 781-786.

21. Guan H, Yin F, Kim JH (2002) Accuracy of inhomogeneity correction in photon radiotherapy from CT scans with different settings. Phys Med Biol 47(17): N223-231.

22. Cozzi L, Fogliata A, Buffa F, Bieri S (1998) Dosimetric impact of computed tomography calibration on a commercial treatment planning system for external radiation therapy. Radiotherapy and oncology 48(3): 335-338.

23. Mathieu KB, Turner AC, Khatonabadi M, McNitt-Gray MF, Cagnon CH, et al. (2013) Varying $\mathrm{kVp}$ as a means of reducing CT breast dose to pediatric patients. Phys Med Biol 58(13): 4455-4469.

24. Yan H, Cervino L, Jia X, and Jiang SB (2012) A comprehensive study on the relationship between the image quality and imaging dose in lowdose cone beam CT. Phys Med Biol 5: 2063-80.

25. Deng J, Chen Z, Yu JB, Roberts KB, Peschel RE, et al. (2012) Testiculardoses in image-guided radiotherapy of prostate cancer. Int J Radiat Oncol Biol Phys 82(1): e39-47.

26. Jones AK, Balter S, Rauch P, Wagner LK (2014) Medical imaging using ionizing radiation: Optimization ofdose and image quality influoroscopy. Med Phys 41(1): 014301. 